\title{
Simultaneous multidopant investigation of rare-earth-doped optical fibers by an ion microprobe
}

\author{
Fotios Sidiroglou, Shane T. Huntington, and Ann Roberts \\ School of Physics, University of Melbourne, Victoria 3010, Australia \\ Richard Stern and Ian R. Fletcher \\ Centre for Microscopy and Microanalysis, M010, The University of Western Australia, 35 Stirling Highway, \\ Crawley, Western Australia 6009, Australia \\ Greg W. Baxter \\ Optical Technology Research Laboratory, Victoria University, P. O. Box 14428, Melbourne, Victoria, 8001, Australia
}

Received July 27, 2006; accepted August 19, 2006;

posted August 24, 2006 (Doc. ID 73454); published October 26, 2006

\begin{abstract}
The relative distribution of five elements present in the core area of several optical fiber samples has been obtained by utilizing nanoscale-secondary ion mass spectrometry. A strong correlation between the rareearth (RE) ion and aluminum was observed, consistent with aluminum's improving the solubility of the RE ion. The central dip in distribution was less severe than that observed for germanium, characteristic of the collapse process during fabrication of the fiber preform. () 2006 Optical Society of America

OCIS codes: $060.0060,060.2410,060.2270$.
\end{abstract}

Rare-earth- (RE) doped fibers constitute a key component in the production of fiber lasers and fiber amplifiers. ${ }^{1}$ These devices have found significant application in a range of scientific and industrial environments. Specifically, in the field of telecommunications they have become the main media for the modulation and amplification of optical signals. As a result, together with the simultaneous growth of the Internet and the demand for higher communication bandwidths, the need to optimize fiber lasers and amplifiers is stronger than ever. Among the many variables vital to the optimal design and operation of such products is an accurate knowledge of the REdopant distribution in the core of the fiber. ${ }^{2-4}$ Also of great importance is the distribution of other elements normally present in the core of RE-doped fibers, since they affect the refractive-index profile of the fiber, which defines the optical properties of the fiber. ${ }^{1}$

To date, two different approaches have been employed to measure the distribution of RE ions in optical fibers. In one of these, various analytical techniques (i.e., secondary ion mass spectroscopy, ${ }^{5}$ electron probe microanalysis, ${ }^{6}$ and extended x-ray absorption fine structure spectroscopy ${ }^{7}$ ) have been applied to fiber preforms from which the fiber is drawn. The information extracted about the preform is then scaled down to match the final fiber dimensions, assuming that there are no changes during the high-temperature drawing process. However, the relationship between the distribution of dopants in the preform and the drawn fiber can be quite complex. As a consequence, there is no guarantee that during fiber drawing the dopant profile of the resulting fiber will be the same as that of the original perform, since it is expected that due to dopant diffusion some redistribution of the RE ions will occur during the draw- ing process. In the second approach, measurements were made directly on the drawn fiber. Transmission electron microscopy (TEM) ${ }^{8}$ Raman microscopy, ${ }^{9}$ and fluorescence-intensity-based confocal microscopy ${ }^{10}$ are some of the techniques previously used for that purpose. More recently, a Raman confocal imaging system has been used for the determination of the Er ion distribution in germano-alumino-silicate optical fibers. ${ }^{11}$

In this Letter, we present the results of the application of a nanoscale-secondary ion mass spectrometry (NanoSIMS) imaging system to determine the elemental distribution in Er- and Yb-doped optical fibers. A full simultaneous multidopant investigation of the fiber core and the adjacent cladding region has been achieved. The relative distribution profiles of the RE (Er or $\mathrm{Yb}$ ), Ge, Al, P, and background Si in the fiber core have been simultaneously acquired with submicrometer resolution. In addition to transverse line scans through the center of the fiber samples, two-dimensional images showing the relative distribution of all the relevant elements present in the fiber core area have also been obtained.

The fiber samples were first mounted in specially designed holders so that the end face of the fibers could be polished to a surface quality of $0.1 \mu \mathrm{m}$ as part of a continuous, flat surface. After polishing, the samples were gold coated to provide surface conductivity. Measurements were performed by using a Cameca NanoSIMS-50 ion microprobe. Chemically reactive primary ions of ${ }^{16} \mathrm{O}^{-}$with impact energies of $16 \mathrm{keV}$ were used to bombard the polished end face of the fiber samples at an angle normal to the sample surface. The resulting sputtered secondary ion beam was then mass analyzed, and the separated beams were recorded by an array of five electron-multiplier 
detectors. Each detector was used to record one of the following species: ${ }^{27} \mathrm{Al}^{+},{ }^{70} \mathrm{Ge}^{+},{ }^{31} \mathrm{P}^{+},{ }^{44}[\mathrm{SiO}]^{+}$, and either ${ }^{166} \mathrm{Er}^{+}$in the case of Er-doped fibers, or ${ }^{174} \mathrm{Yb}^{+}$, in the case of $\mathrm{Yb}$-doped fibers. As a result, simultaneous acquisition of all dopants present in the central fiber core area was achieved.

The choice of the isotopes to record for $\mathrm{Er}$ and $\mathrm{Yb}$ was relatively simple, because each fiber core has a single RE dopant, and the cladding is theoretically $\mathrm{RE}$ free. $\mathrm{RE}$ oxide interferences are therefore insignificant, and the most abundant isotopes of $\mathrm{Er}$ and $\mathrm{Yb}$ were used. No significant interference peaks were observed in the relevant mass ranges, and element identities were confirmed by measuring $\mathrm{Er}$ and $\mathrm{Yb}$ isotope ratios. The mass spectrum around Ge is more complicated, mainly because of the presence of various $\mathrm{Si}_{2} \mathrm{O}^{+}$species. These were avoided by recording ${ }^{70} \mathrm{Ge}^{+}\left(20 \%\right.$ of total $\mathrm{Ge}$ ), which avoids $\mathrm{Si}_{2} \mathrm{O}^{+}$and is resolved from a small $\mathrm{Al}_{2} \mathrm{O}^{+}$peak at the working mass resolution.

During acquisition, there was no attempt to use external standards for abundance calibration, since the important parameters investigated here are the distributions in the cross section. Therefore all data for each element are internally scaled. In addition, data are first normalized to $\mathrm{SiO}^{+}$to remove minor distortion caused by uneven secondary ion extraction from the analyzed area of the sample. Image data are then independently displayed on a continuous gray scale for each element. For the line scans, data for each element were normalized to the highest signal recorded for that element to facilitate plotting.

Prior to image acquisition, the sample area under investigation was rastered by using a large-diameter primary ion beam so that part of the gold coating was removed, and secondary ion emission was maximized through oxygen implantation. Beam-raster images were recorded within the implanted area by using a primary ion probe with a diameter of $\sim 0.4 \mu \mathrm{m}$, but which was slightly elliptical because of ion optic aberrations. The primary ion current for data acquisition was $(\sim 1 \mathrm{pA})$. Line scans were acquired by using beam steering. After recording a low-quality (fast) image, the scan trace was defined electronically (onscreen), and the primary beam was stepped along the trace under software control. This was repeated for reasons analogous to imaging: for the first scan, a broad, high-intensity primary ion beam was used for gold removal and $\mathrm{O}^{-}$implantation, and the fine probe was used for data acquisition. Provided the element distributions are reasonably symmetric, line scans give better detail than images because of the longer integration time available for each pixel.

Typical cross-section images for four elements present in the core of an Er-doped fiber amplifier (EDFA) sample are shown in Fig. 1. With the exception of the $\mathrm{Er}^{+}$, for which the secondary ion signal was weak under the acquisition conditions, the distribution of elements in the core of the fiber and the adjacent inner cladding are well defined. In the case of $\mathrm{P}$, deposition rings defining the inner cladding are clearly visible. The apparent asymmetry in Fig. 1(d) is a consequence of the ellipticity of the primary ion beam discussed earlier. All subsequent line scans were recorded at approximately the angle shown by the arrow in Fig. 1(a)

A line scan through the center of an experimental Er-doped fiber with a relatively high Er concentration $\left(\sim 2000\right.$ parts in $\left.10^{6}\right)$ is shown in Fig. 2. This fiber was fabricated by using the modified chemicalvapor deposition (MCVD) technique, while the Er and $\mathrm{Al}$ were incorporated by solution doping. It is known that during the incorporation of $\mathrm{Ge}$ and $\mathrm{P}$ into optical fibers, as a consequence of their volatile nature at high temperatures, it is possible that depletion and a corresponding refractive-index depression can be produced during the collapse of the MCVD. A similar behavior has been reported for the incorpora-
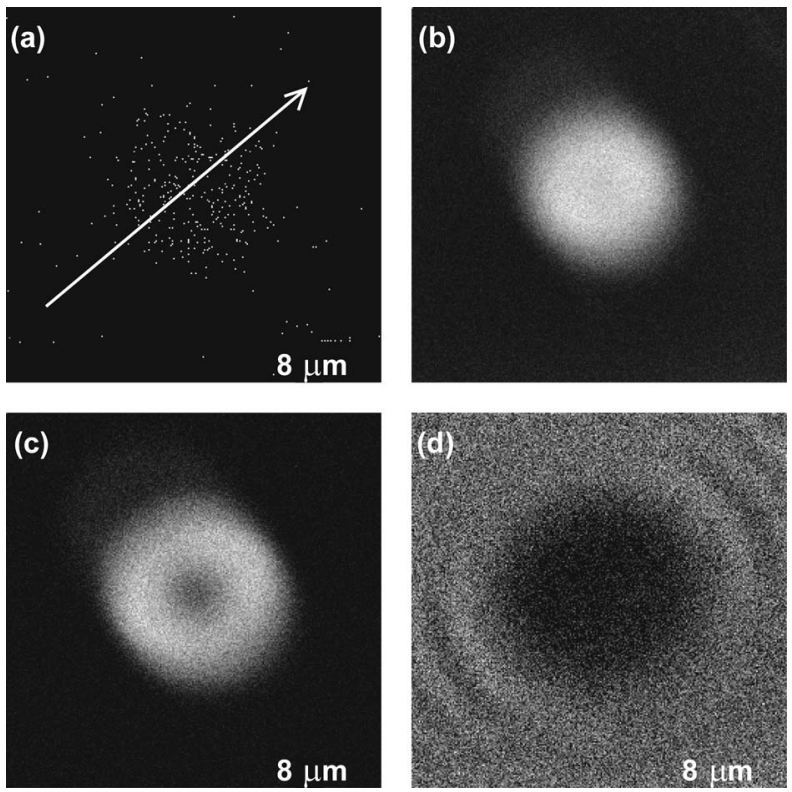

Fig. 1. Cross sections of the relative ion distribution in the core of an EDFA sample. (a) Er, (b) Al, (c) Ge, (d) P. The arrow indicates the direction along which subsequent line scans were obtained.

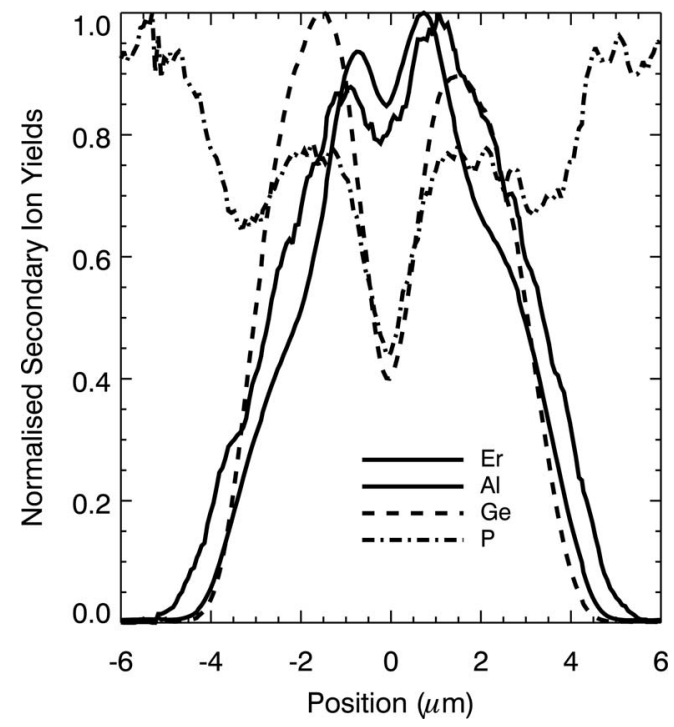

Fig. 2. Transverse line scan through the center of an experimental Er-doped fiber, showing the relative ion distribution of all dopants present in the fiber core. 


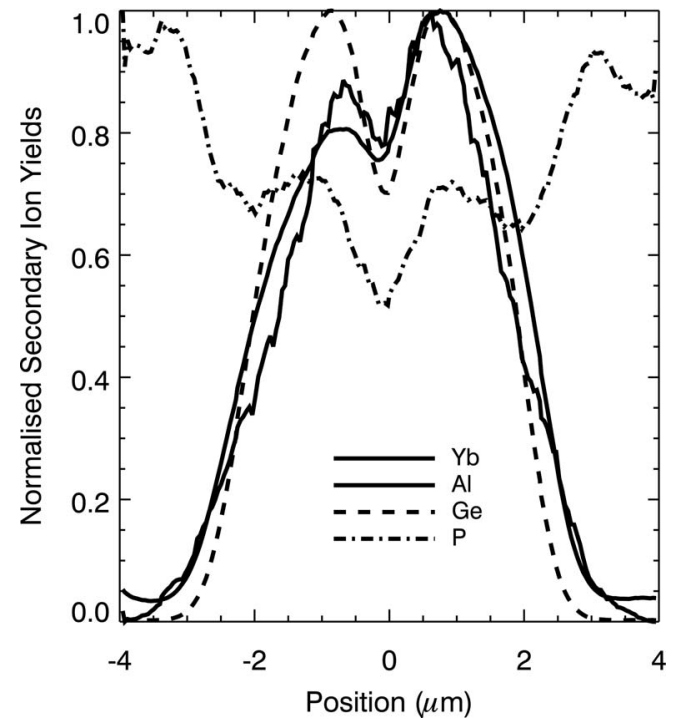

Fig. 3. Transverse line scan through the center of an experimental Yb-doped fiber, showing the relative ion distribution of all dopants present in the fiber core.

tion of Er in a germano-silicate host, ${ }^{12}$ while no depletion of the Er in the center was observed in the presence of $\mathrm{Al}{ }^{13}$ Figure 2 shows that indeed both Ge and $\mathrm{P}$ exhibit significant depletion within the central core. However, so do both Er and Al, although not to the same extent. This behavior of both $\mathrm{Al}$ and $\mathrm{Er}$ in this fiber could be a direct outcome of the relatively high concentration of the RE element. In this case, it can also be seen that the Er distribution follows that of Al. Similar behavior of Er was detected in all of the investigated fiber samples.

In the case of the $\mathrm{Yb}$-doped fibers, $\mathrm{Yb}$ follows the distribution of $\mathrm{Al}$ in a way similar to Er, as displayed in Fig. 3. This fiber was also fabricated by the MCVD and solution doping techniques. The $\mathrm{Yb}$ distribution clearly follows that of $\mathrm{Al}$, while the presence of $\mathrm{Al}$ again does not prevent $\mathrm{RE}$ element depletion in the center core area.

In conclusion, a comprehensive analysis of the relative ion distribution of five elements present in the fiber core area of various RE-doped fiber samples was obtained by utilizing a commercial NanoSIMS microscope. All elements were simultaneously detected and analyzed from the end face of optical fibers with an estimated spatial resolution of $\sim 0.4 \mu \mathrm{m}$. Valuable information regarding the solubility and diffusion characteristics of various elements typically present in the core of RE-doped optical fibers was gathered. Although incorporation of $\mathrm{Al}$ improves the solubility of the RE, it does not prevent the RE concentration from being depressed along the central core region. The apparent correlation between the $\mathrm{Al}$ and $\mathrm{RE}$ profiles suggests that aiming toward a Gaussian distribution for Al during the fabrication process will ensure that the RE will not be depleted in the fiber core center. Information of this kind can provide feedback into fabrication techniques to improve the design and operation of fiber lasers and amplifiers and permit more precise modeling of fiber performance.

The authors thank J. Canning of the Optical Fiber Technology Centre (OFTC), Australia, T. Nguyen of Victoria University, and Laboratoire de Physique de la Matière Condensée, Nice for the provision of the fiber specimens. This work is sponsored in part by the Australian Research Council. We also acknowledge the technical, scientific, and financial assistance from the Nanostructural Analysis Network Organisation-Major National Research Facility. F. Sidiroglou thanks the Victoria state government (ICT scheme) and the Science Faculty at the University of Melbourne for partly funding his Ph.D. candidature F. Sidiroglu's e-mail address is fotios @ph.unimelb.edu.au.

\section{References}

1. M. J. F. Digonnet, Rare-Earth-Doped Fiber Lasers and Amplifiers, 2nd ed. (Dekker, 2001).

2. E. Desurvire, J. L. Zyskind, and C. R. Giles, J. Lightwave Technol. 8, 1730 (1990).

3. W. L. Barnes, R. I. Laming, E. J. Tarbox, and P. R. Morkel, IEEE J. Quantum Electron. 27, 1004 (1991).

4. J. C. Martin, Opt. Commun. 194, 331 (2001).

5. N. Kagi, A. Oyobe, and K. Nakamura J. Lightwave Technol. 8,1319 (1990).

6. T. Kashiwada, M. Shigematsu, T. Kougo, H. Kanamori, and M. Nishimura, IEEE Photon. Technol. Lett. 3, 721 (1991).

7. D. T. Bowron, R. J. Newport, J. S. Rigden, E. J. Tarbox, and M. Oversluizen, J. Mater. Sci. 38, 485 (1996).

8. A. C. Pugh, R. P. Stratton, and D. B. Lewis, J. Mater. Sci. 29, 1036 (1994).

9. S. Rogard, A. Bulou, H. Poignant, and M. Rousseau, Opt. Mater. 4, 557 (1995).

10. A. Othonos, J. Wheeldon, and M. Hubert, Opt. Eng. 34, 3451 (1995).

11. F. Sidiroglou, S. T. Huntington, A. Roberts, and G. W. Baxter, Opt. Express 13, 5506 (2005).

12. B. J. Ainslie, J. R. Armitage, S. P. Craig, and B. Wakefield, in Proceedings of IEE 14th European Conference on Optical Communication (Institution of Electrical Engineers, 1988), pp. 62-65.

13. B. J. Ainslie, J. Lightwave Technol. 9, 220 (1991). 